\title{
Design and Simulation of the Wireless Communication Protocol applied to Small Satellite
}

\author{
QI QI \\ Research center of Satellite Technology \\ Harbin Institute of Technology \\ Harbin, China \\ e-mail: lyqq1991@163.com \\ Li Peng-fei \\ Research center of Satellite Technology \\ Harbin Institute of Technology \\ Harbin, China \\ e-mail:lpf_365@163.com
}

\author{
XU Guo-dong, \\ Research center of Satellite Technology \\ Harbin Institute of Technology \\ Harbin, China
}

\begin{abstract}
Wireless communication technology between subsystems in satellite can be effective measures for the needs of the development of small satellite system, which has the advantage of fast testing, fast integration and fast assembling. It makes up the existing defect of traditional satellite which used traditional wired bus. Paper analyzed the link performance of intra-satellite networking and the relationship between BER and SNR; discussed modulation, demodulation method and transmit power and receiving sensitivity for each model. A wireless network protocol model based on dynamic slots distribution has been designed according to the international OSI model including Physical layer, Data link layer and Application layer, and verified the basic function and plug and play function of the proposed protocol of intra-satellite wireless networking system by constructing a networking system composed of $\mathbf{3 0}$ nodes. The results show that protocol is not only versatile, reliable but also has the function of plug and play.
\end{abstract}

Keywords-Intra-satellite; Channel nalysis; Communication protocol; Plug and play

\section{INTRODUCTION}

United States launched the "tactical satellite program (TacSat series)" in 2004, in order to focus on the development of small satellites that have short development cycle and high launch speed [1]. In order to satisfy the needs in the development of fierce competition in space, research on the small satellite platform which can do rapid test, integration and assembly is of great significance. The complex and cumbersome wired communication cables and interfaces of Intra-satellite become barriers for the development of small satellites.Table1 shows the proportion of some typical domestic and foreign satellite connection cable and interface. Connecting the subsystems to a bus, the traditional structure of the satellite forms a single point, posing a potential threat to the safety of the satellite. Given this situation, the experts at the University of Delft proposed the idea to apply RF wireless technology to in satellite communications to replace traditional wired bus on the 57th International Astronautical Congress in 2006, the wireless network technology applied to the inside spacecraft have brought more and more attention in the last two years. University of Surrey and University of Delft proposed to use commercial application protocol directly on in satellite wireless communications [3-5]. And some proposed to realize wireless communication in satellite based on infrared communication [6].Although using the existing commercial protocols can meet the needs of wireless communication in satellite, the commercial protocols have the defects of complicated, large-power, low real-time. Using infrared communication don't have to avoid collision issues by protocol, nevertheless, it constrained the design of the star structure greatly [7].

Paper presents special wireless satellite platform internal network system protocol, not only meets the demand for high reliability and real-time of aerospace, but also makes the network with the function of automatic restructure and plug and play.

TABLE I. PLUG IN INTERFACE AND A CONNECTING CABLE FOR SATELLITE GRAVITY

\begin{tabular}{|c|c|c|c|}
\hline satellite & Net weight & Cable & rate \\
\hline Goce & $740 \mathrm{~kg}$ & $60 \mathrm{~kg}$ & $8 \%$ \\
\hline Cluster- 2 & $540 \mathrm{~kg}$ & $33.4 \mathrm{~kg}$ & $6.2 \%$ \\
\hline MarsExpress & $450 \mathrm{~kg}$ & $28 \mathrm{~kg}$ & $6.2 \%$ \\
\hline Smart-1 & $280 \mathrm{~kg}$ & $22.1 \mathrm{~kg}$ & $8 \%$ \\
\hline Proba & $100 \mathrm{~kg}$ & $7.6 \mathrm{~kg}$ & $7.6 \%$ \\
\hline
\end{tabular}

II. PHYSICAL LAYER OF WIRELESS COMMUNICATION TECHNOLOGY IN SATELLITE

The physical layer is at the bottom of OSI reference model, it is responsible for providing a connection for the transmission of original bit stream of data link layer. 


\section{A. Selection for Modulation and demodulation mode}

Modulation and demodulation method has great effect on BER and performance of network communication system. Common methods include frequency modulation (FM), amplitude modulation (AM) and phase modulation (PM). In the course of in satellite transmission, AM method is susceptible to channel, amplifier and the distance between two points, distort the signal envelope packet, thus increases the BER. Propagation of electromagnetic waves have severe multipath phenomena corresponding to the small satellite cabin,, phase modulation increases BER due to superposition of multiple reflected waves. Frequency modulation has no such problems above, so fits wireless network communications in satellite better

\section{B. Analysis on wireless transceiver unit performance of in satellite}

Wireless communication is not as reliable as wired communication, so people have to increase the allowance of communication channel to improve reliability.

The allowance of communication link affect by transmission power, receive sensitivity and spatial fading. Spatial fading is fixed as the relatively fixed installation position of satellite subsystems in the internal satellite, the communication link should mainly considerate the effect of transmission power and reception sensitivity.

To reduce power consumption and complexity of the radio transceiver unit, the transmission power is usually close to $0 \mathrm{~dB}$. Acceptable sensitivity should satisfy (1):

$$
\begin{aligned}
P_{\min } & =P_{N}+P_{t h}+P_{l}+P_{0} \\
& =10 \log \left(k T_{s} R\right)+P_{t h}+P_{l}+P_{0}
\end{aligned}
$$

Where $P_{\min }$ is reception sensitivity, $P_{N}$ is noise power, $P_{t h}$ is demodulation SNR threshold, $P_{l}$ is loss, $P_{0}$ is noise figure, units are $\mathrm{dB} ; k$ is Boltzman constant, $T_{s}$ is noise temperature, $R$ is communication rate.

From (1), when $P_{t h} 、 P_{l} 、 P_{0}$ is determined, reception sensitivity only relates to communication rate. As for the subsystems in satellite, transmission information includes command parameters, experimental data and satellite status information; transmission rate at $100 \mathrm{Kbps}$ can satisfy the needs for system work. Considering design margin, the communication rate should be greater than $250 \mathrm{Kbps}$ at least.

Typically, cable loss, demodulation loss, and transmission path loss etc., can add up to $5 \mathrm{~dB}$ to $7 \mathrm{~dB}$, if FSK demodulation threshold is $14.6 \mathrm{~dB}$, usually select $1 \mathrm{~dB}$ for noise factor, $300 \mathrm{~K}$ for $T_{s}$. Put the above parameters and communication rate at $250 \mathrm{Kbps}$ together into (1), the receiver sensitivity is about $-95 \mathrm{dBm}$ at the rate of $250 \mathrm{Kbps}$, taking the actual processing of the RF communication module into account, communication unit of a wireless communication elementary unit should choose RF communication module, whose rate is greater than $250 \mathrm{Kbps}$ and sensitivity is not less than $-90 \mathrm{dBm}$.

\section{MAC LAYER DESIGN}

MAC is at the second layer of OSI reference model, providing reliable data transmission on the basis of the physical layer according to certain media access control policy, which includes the establishment and maintenance of communication between each node, and synchronize the session.

\section{A. Frame structure design}

Considering the utilization efficiency of channel, use fixed-length as frame structure to save identifier at the end of frame, the frame structure consists of three parts.

Set central control computer in satellite as master node, there are three types of frame structure: network starting frame, slot allocation frame and synchronous frame, distinguished by frame identifier segments. Set other subsystems as a sub-node, including slots request frame and response frame. Fig. 1 shows the three combinations of frame, the network start frame used for starting the whole network, issued by host computer, lower computer send the communication response frame in accordance with bus communication system in 2.2 after receiving the frame. Master node uses slot allocation frame to allocate communication slot for sub nodes. Each communication cycle starts from synchronous frame; sub-nodes reset their timer after receiving this frame. Sub-node sends a request frame when it has not got the slot allocated from master node.

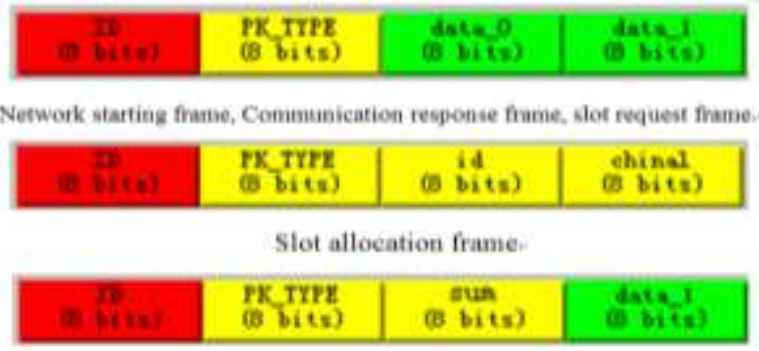

Synchronous frame.

Figure 1. Frame type

\section{B. Sharing wireless bus communication mechanism}

A variety of measures are used to solve collisions, the most common way is Carrier Sense Multiple Access/ Collision Detection (CSMA/MA), but in the satellite control system, CSMA/MA cannot control packet transmission delay, which increases the difficulty in satellite attitude orbit control algorithm, and packet loss rate increases under the large-scale network accordingly. In order to meet satellite internal communication requirement, as is shown in Fig.2, protocol divides each communication cycle into a number of time slots, each node occupies one or more slots and only sends packets in its own time slot, this approach not only fix the network packet transmission delay, but also solve the problem of collision. 


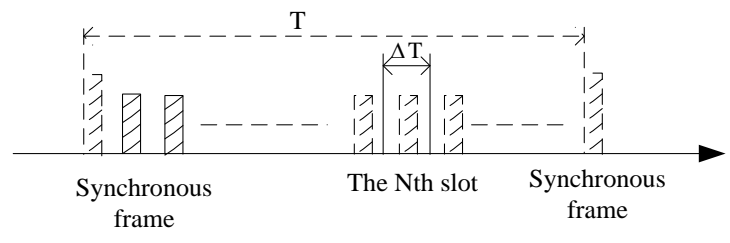

Figure 2. Time sharing communication mechanism

\section{Dynamic slots allocation of Ad hoc network mechanism \& plug and play mechanism}

After powering the entire satellite, slave nodes keep silent, master node starts over, sends network start frame, then slave node sends slot request frame after receiving it at random intervals in a communication cycle, the random interval is an integral multiple of the slot $\Delta \mathrm{T}$. If master node receives the slot request frame successfully, the allocated slot and ID number would be record in its network table, then send slot allocation frame to slave nodes. While if master node missed the request due to collision, the slave node would send slot request frame in the next communication cycle until it received the slot allocation frame sent by master node. When at least one slave node is allocated slot successfully, master node send synchronous frame with $\mathrm{T}$ as period.

After master node sending synchronous frame, network works normal, as is shown in fig.1, the total node number of the sum segment in synchronous frame is $N$. Consider that one node was not allocated slot, when it receive the synchronous frame sent by master node, send slot request frame at a random time period between $\Delta T \times N+T_{0}$ and $T$ to make sure that would not affect the communication of nodes which already in the network. This mechanism can solve the problem of dynamic slot allocation and satisfy plug and play as well.

If master node has not received the appropriate response of certain slave node in two consecutive communication cycles; it will withdraw the time slot correspondingly, and assign it to newly joined node as priority.

\section{Ad hoc network time analysis}

The above shows dynamic slot allocation Ad hoc network mechanism is a process from chaos to stability. The communication between subsystems in satellite cannot guarantee normal in the process of Ad hoc network, thus we have to analysis the networking time.

Set throughput $S$ represents the average number of successfully sent frames in the transmission time, the network load $G$ is the average frames within frame delivery time, including successfully sent frames and unsent ones due to collision

The relationship between the throughput $S$ and network load $\mathrm{G}$ is $S=P G$ under steady state, where the probability of successfully sent frames is $P$ :

$$
\begin{aligned}
& P=P\left(\text { the interval of arrival time }>T_{0}-T_{\mathrm{x}}\right) \\
& \times P\left(\text { the interval of arrival time }>T_{\mathrm{x}}\right)
\end{aligned}
$$

Where $T_{0}$ is the time for one frame transmit in the channel, assuming that the transmission start time of the frame in the channel follows Poisson distribution[8], the probability density of arrival time interval can be expressed as (2):

$$
a(t)=\frac{G}{T_{0}} e^{-\frac{G}{T_{0}} t}
$$

$P$ can be written as follows:

$$
P=\int_{T_{0}-T_{X}}^{\infty} a(t) d t \int_{T_{x}}^{\infty} a(t) d t
$$

That is

$$
P=e^{-G}
$$$$
\text { So the throughput } \quad S=G e^{-G}
$$

According to (5), when $G=1, S_{\max }=0.3679$.

Assume that there are $\mathrm{n}$ nodes are to add to network, the 1 st slot can add $a_{1}=n S_{\max }$ nodes to the network at the maximum probability, the 2nd slot can add $a_{2}=\left(n-a_{1}\right) S_{\max }=n-\left(n-n S_{\max }\right) S_{\max }$ nodes to network at the maximum probability, as for the mth slot,

$$
a_{m}=\left(n-\sum_{i=1}^{m-1} a_{i}\right) S_{\max }
$$

So after the $\mathrm{m}^{\text {th }}$ slot, the number of nodes joined the network is:

$$
Y(m)=\sum_{i=1}^{m} a_{i}
$$

Put (6) into (7), assume that the number of nodes joined network is $Y(m)$, thus

$$
Y(m)=\sum_{i=1}^{m}\left(n-\sum_{j=1}^{i-1} a_{j}\right) S_{\max }
$$

From (8), when $m>8, Y$ tends to $n$, after 8 slots, new nodes almost add to network successfully.

\section{SimUlation AND VERIFICATION}

Paper uses OPNET to simulate the protocol , according to 3, establishes finite state machine between master node and slave nodes respectively. Decompose the action into three steps: receive frame, parse frame, and transmit frame. Node sets the arrived frame as a trigger, then returns to wait state after three actions.

Assume that there are 30 sub nodes, 1 master node, set communication cycle $\mathrm{T}$ as $500 \mathrm{~ms}$, time slot as $5 \mathrm{~ms}$. Simulation shows in Fig.3, simulation data statistic from master node. The blue line is the number of normal communication nodes in current network, red line is the sub-node's ID occupied the wireless channel in current network, seen from Fig. 3 we can see that the number of normal communication nodes is 30 after $3 \mathrm{~s}$, which is all nodes have joined network, and network works stable in the last 20s. Fig.4 and Fig.5 are the subfigure of Fig.3, represent the stage of Ad hoc network-the process of node joining work, and a cycle in normal working process respectively. Compare Fig.4 and Fig.5, no interference caused by the new joined nodes in the stage of Ad hoc network.

Almost every satellite subsystems has host machine and preparation machine, to verify the robustness of this protocol, the master node switch machine at $8 \mathrm{~s}$ moment, the 13th sub node switch machine at $15 \mathrm{~s}$ moment, the simulation result is shown in Fig.6. As can be seen from the figure, whether master node or sub node switches the 
master and preparation machine, the network can return normal in a short time.

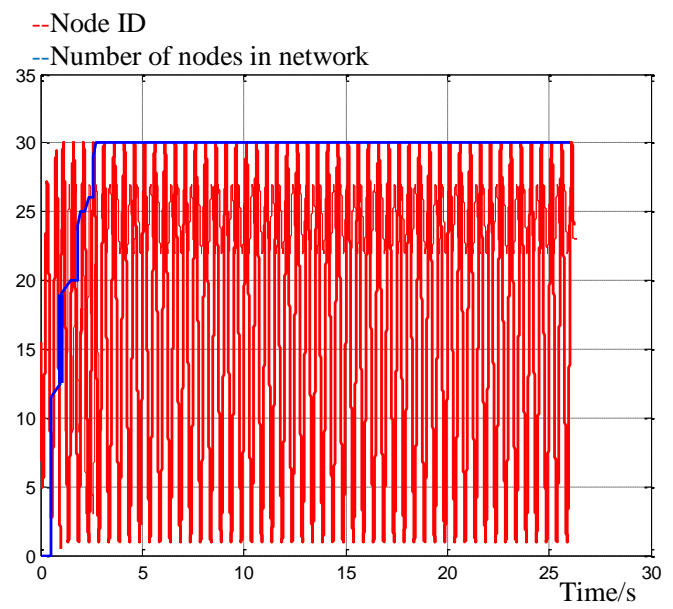

Figure 3. The process of node joining the network

--Node ID

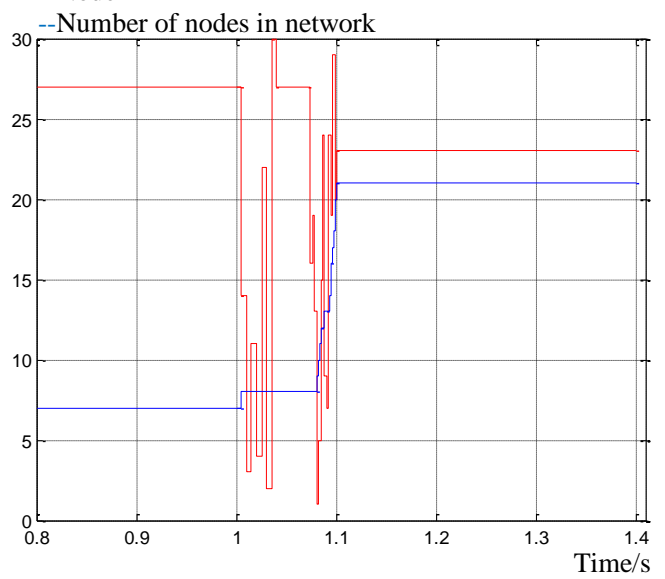

Figure 4. The process of node joining the network

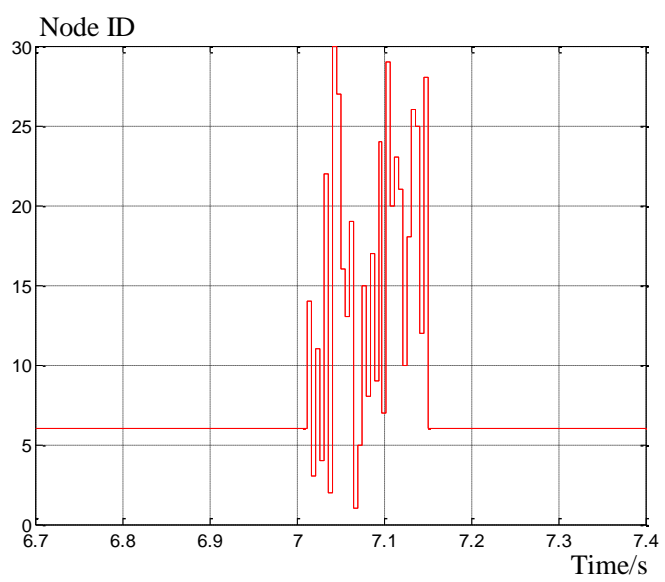

Figure 5. A cycle in normal working process

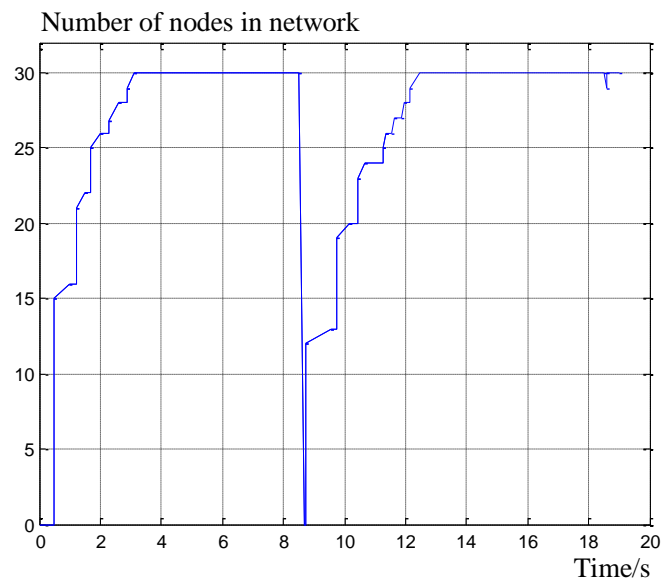

Figure 6. Network restoration after nodes' failure

\section{CONCLUSIONS}

Paper analyzed and reached the conclusion that when the transmitter power is $0 \mathrm{dBm}$, the receiver sensitivity is $90 \mathrm{dBm}$, the RF modulation and demodulation mode adapted to wireless network satellite platform is FSK or GFSK. According to the actual needs of the satellite work, designed and established a wireless network communication protocol based on dynamic slot allocation technique, and verified by simulation. Simulation results show that this protocol is not only versatile, reliable but also has the function of plug and play. The time of all nodes add to the entire internal satellite network is no more than $5 \mathrm{~s}$.

\section{REFERENCES}

[1] Mission need statement for operationally responsive spacecraft. Report of Air Force Space Command (AFSPC). 2001: 103-112.

[2] J. Lyke, S. Cannon, D. Fronterhouse. A Plug-and-play System for Spacecraft Components Based on the USB Standard[C]. proceedings of the 19th Annual AIAA/USU Conference on Small Satellites, Logan, UT, August, 2005, 96-101.

[3] M. Martin, D. Fronterhous, J. Lyke. The implementation of a Plugand-Play satellite bus[C]. Proceedings of the 22nd Annual Comference on Small Staellites, Chicago, USA, June 2008: 311314

[4] R. Amini, G. Aalbers, R. Hamann. New generations of spacecraft data handling systems: Less harness, more reliability[C] Proceedings of 57th International Astronautical Congress, Acta, Astronautica September 2006,231-237.

[5] J. Rotteveel, G.T. Aalbers, A.R. Bonnema. Delfi-C3: a Student Nanosatellite Test-bed for in-orbit Demonstration of Micro Systems Technology[J]. Delft University of Technology, G.L.E. 2008: 13-17.

[6] Remo Tamayo, Jose Alonso. Optical wireless links for IntraSatellite communications: reflection models and hardware optimization[J]. Journal Of Aerospace Computing, Information, and Communication, 2010,7:118-133.

[7] Cai Ran, Xue Cai, Cao Jie, Fu Jin, Hu Yu. The intra satellite wireless optical network communication technology and its realization[J]. Acta Photonica Sinica, 2004, 34(2): 113-119.

[8] Huang Yongfeng,Li Xing.Computer network tutorial[M].Beijing : Tsinghua University, 2006:99-102. 